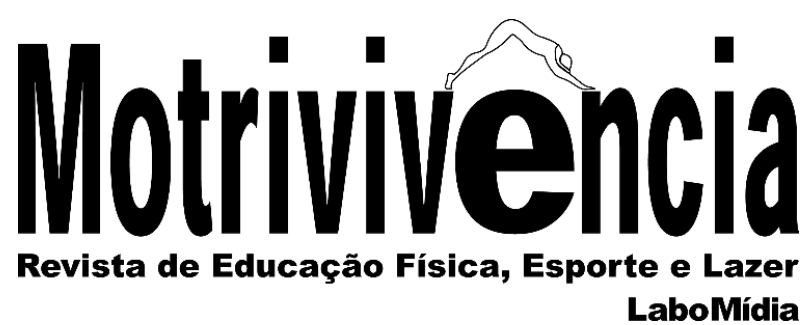

\title{
Modelos e abordagens de implementação para a análise de políticas de esporte e lazer: construção do grupo de pesquisa Polis/NeoPolis
}

\section{RESUMO}

Os avanços do campo de análise de políticas públicas no Brasil ocorreram a partir dos anos 2000 e sua materialização se dá em diferentes áreas disciplinares. A partir de 2010, observa-se um recrudescimento da produção científica na área de políticas públicas de esporte e lazer com um esforço de aproximação do arcabouço teórico das ciências sociais. O objetivo do ensaio foi apresentar o grupo de pesquisa Polis/NeoPolis da UFMG e refletir sobre a literatura do campo das políticas públicas que tem fundamentado seus trabalhos e publicações na área do esporte e lazer. $\mathrm{O}$ grupo tem investido em estudos de políticas públicas com o intuito de produzir conhecimento acerca de uma política setorial que coadune com uma produção mais ampla do campo de políticas públicas nacionais e internacionais. Acredita-se que o recorte escolhido sobre os estudos de implementação possa contribuir com as pesquisas desenvolvidas na área de esporte e lazer.

PALAVRAS-CHAVE: Implementação; Políticas públicas; Esporte e lazer

\begin{tabular}{|c|}
\hline 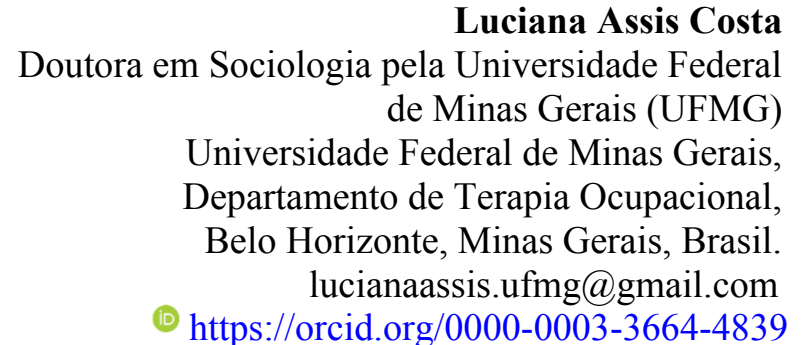 \\
\hline
\end{tabular}

Luciano Pereira da Silva

Doutor em Educação pela Universidade Federal de Minas Gerais (UFMG)

Universidade Federal de Minas Gerais,

Departamento de Educação Física,

Belo Horizonte, Minas Gerais, Brasil. lpereira45@hotmail.com

@ $\underline{\text { https://orcid.org/0000-0003-3456-5766 }}$

Daniete Fernandes Rocha

Doutora em Sociologia pela Universidade Federal de Minas Gerais (UFMG)

Faculdade Arnaldo Janssen, Departamento de Medicina Veterinária Belo Horizonte, Minas Gerais, Brasil. daniet.rocha@gmail.com

https://orcid.org/0000-0002-5624-5459

Brisa de Assis Pereira

Doutoranda em Estudos do Lazer pela Universidade Federal de Minas Gerais (UFMG)

Universidade Federal de Minas Gerais, Programa de Pós-Graduação Interdisciplinar em

Estudos do Lazer,

Belo Horizonte, Minas Gerais, Brasil.

Brisadeassis@hotmail.com

๑https://orcid.org/0000-0002-8647-7971 
Natascha Stephanie Nunes Abade Doutoranda em Estudos do Lazer pela Universidade Federal de Minas Gerais (UFMG)

Universidade Federal de Minas Gerais, Programa de Pós-Graduação Interdisciplinar em

Estudos do Lazer,

Belo Horizonte, Minas Gerais, Brasil. natascha_abade@yahoo.com.br

-https://orcid.org/0000-0002-7393-546X

Marcelo de Melo Mendes

Doutorando em Estudos do Lazer pela Universidade Federal de Minas Gerais (UFMG)

Universidade Federal de Minas Gerais, Programa de Pós-Graduação Interdisciplinar em

Estudos do Lazer,

Belo Horizonte, Minas Gerais, Brasil. marcelomm@pbh.gov.br

(- https://orcid.org/0000-0001-6227-0661

Marcus Peixoto de Oliveira

Mestre em Estudos do Lazer pela Universidade

Federal de Minas Gerais (UFMG)

Universidade Federal de Minas Gerais,

Programa de Pós-Graduação Interdisciplinar em

Estudos do Lazer,

Belo Horizonte, Minas Gerais, Brasil. po.marcus@gmail.com

는 https://orcid.org/0000-0002-8632-2961 
Implementation models and approaches for the analysis of sports and leisure policies: the construction of Polis/NeoPolis research group

\begin{abstract}
Since the 2000s, the studies on Public Policy (PP) Analysis advanced and its materialization takes place in different areas. From 2010, there has been an increase of the scientific production in Sport and Leisure PP area, with an effort to bring the theoretical framework of the Social Sciences closer to the discussion. The aim of this essay was to present the research group Polis/NeoPolis from UFMG and reflect about the literature in the PP field that has been supporting the group's works and publications in the area of Sport and Leisure Studies. The group has invested in studies about PP in order to promote the knowledge production about a sectional policy that matches with a broader production in the national and international PP field. It is believed that the chosen subject about the Studies of Implementation can contribute with the research to be developed in the Sport and Leisure field.
\end{abstract}

KEYWORDS: Implementation; Public policy; Sport and leisure.

Modelos de implementación y enfoques para el análisis de políticas deportivas y de ocio: construcción del grupo de investigación Polis/NeoPolis

\title{
RESUMEN
}

Los avances en el campo del Análisis de Políticas Públicas (PP) en Brasil ocurrieron desde la década del 2000 y su materialización se da en diferentes áreas disciplinarias. A partir de 2010 se ha producido un incremento de la producción científica en el ámbito de las PP de deporte y ocio con un esfuerzo por acercar el marco teórico de las ciencias sociales. El objetivo del ensayo fue presentar el grupo de investigación Polis/NeoPolis de la UFMG y reflexionar sobre la literatura en el ámbito de las políticas públicas que viene apoyando sus trabajos y publicaciones. El grupo ha invertido en estudios de PP con el fin de producir conocimiento que sea consistente con una producción más amplia en el campo de las políticas públicas nacionales e internacionales. Se cree que el apartado elegido sobre los estudios de implementación puede contribuir a la investigación desarrollada en el área.

PALABRAS-CLAVE: Implementación; Políticas públicas; Deportes y ocio. 


\section{INTRODUÇÃO}

Os avanços em prol da consolidação do campo de análise de políticas públicas no Brasil ocorreram a partir dos anos 2000 e sua materialização tem se dado por meio de pesquisas desenvolvidas em diferentes áreas disciplinares. Essa característica multidisciplinar permite uma compreensão ampla de um fenômeno extremamente complexo, contudo resulta em uma especialização setorializada e, amiúde, na fragmentação do conhecimento produzido no país sobre a área, que se reflete em um gap teórico (BRASIL \& CAPELLA, 2016).

Em um estudo de revisão foram analisados cento e quinze artigos que abordavam o tema política pública entre os anos de 2002 e 2014, e identificadas dezenove políticas setoriais como alvo principal de análise. As áreas setoriais com maiores frequências constatadas foram a da saúde (20\%), seguida por educação (13\%), participação (12\%) e assistência social (11\%) (SUDANO, SOARES \& VERGILI, 2015). Nesse mesmo sentido, segundo Brasil e Capella (2016), o estudo sobre políticas públicas tem avançado em vários campos de conhecimento, em distintos departamentos de diferentes instituições de ensino do país.

No caso da área de esporte e lazer, os debates sobre as políticas públicas se ampliaram a partir da década de 1990, quando o esporte e o lazer obtiveram maior notoriedade nas pautas políticas, uma vez que passaram a ser considerados direitos fundamentais pela Constituição Federal de $1988(\mathrm{CF} / 88)$.

A partir dos anos 2010, observa-se um recrudescimento da produção científica na área de políticas públicas de esporte e lazer com um esforço de aproximação do arcabouço teórico das ciências sociais. Um levantamento realizado nas bases de dados disponíveis no portal CAPES, no período de 2015 a 2020, identificou 120 artigos nacionais que abordavam o tema políticas públicas de esporte e lazer, sendo 80 artigos específicos sobre implementação, o que demonstra a expansão da área.

Na UFMG, em 2006, foi implantado o Programa de Pós-graduação Interdisciplinar em Estudos do Lazer, vinculado à Escola de Educação Física, Fisioterapia e Terapia Ocupacional (EEFFTO). Uma das linhas de pesquisa do programa é a de Formação, Atuação e Políticas de Lazer. Atualmente nove docentes estão vinculados a essa linha, e uma das estratégias de fortalecimento do campo nesta área foi a criação do grupo de pesquisa Polis e o NeoPolis, atualmente integrados.

O referencial teórico que tem sustentado as reflexões e discussões do Grupo Polis/NeoPolis da UFMG está apoiado no campo de análise de políticas públicas, e nas contribuições, sobretudo, 
das áreas da ciência política e da sociologia. O campo de políticas públicas é considerado uma subárea da ciência política que tem como foco a investigação sobre a ação do estado, ou seja, o modo como o Estado se posiciona e produz as políticas (BRASIL \& CAPELLA, 2016). Assim, o campo se detém na investigação sobre "políticas e programas governamentais, particularmente suas condições de emergência, seus mecanismos de operação e seus prováveis impactos sobre a ordem social e econômica" (ARRETCHE, 2003:02).

O objetivo deste ensaio foi apresentar o grupo de pesquisa Polis/NeoPolis da UFMG e refletir sobre o arcabouço teórico do campo das políticas públicas que tem fundamentado os trabalhos e publicações do grupo na área do esporte e lazer. Optou-se, para este estudo, pelo recorte na temática de implementação de políticas públicas, à luz da quarta geração de estudos da área. Busca-se, a partir desse referencial teórico, uma análise mais abrangente dos temas específicos da área do esporte e lazer, na tentativa de contribuir para a redução do gap evidenciado na produção científica setorializada, não apenas na área do esporte e lazer, mas nas demais políticas setoriais.

Os estudos sobre implementação foram objeto de investigações que procuraram oferecer novas explicações sobre esse processo, seja em relação ao debate teórico, seja por meio de reflexões sobre sua prática no Brasil (FARIA, 2012; CAVALCANTE \& LOTTA, 2015). Constituem-se como uma vertente do campo de análise de políticas públicas que busca compreender o momento específico da materialização ou concretização das políticas (SOUZA, 2003; LOTTA, 2019).

Lotta (2019) nos chama atenção para o fato de que grande parte de estudos que utiliza o termo "implementação" tem abordado a temática de forma metafórica, como um momento ou parte da política pública, ou como um sinônimo de execução, e não como um objeto analítico. No grupo de pesquisa Polis/NeoPolis, temos investigado as políticas públicas de esporte e lazer no seu sentido abrangente, apoiado no referencial teórico do campo de análise de políticas públicas.

\section{Breve apresentação do grupo de pesquisa Polis/NeoPolis da UFMG}

O surgimento e o desenvolvimento do Polis/NeoPolis ${ }^{1}$ estão diretamente relacionados ao Programa de Pós-Graduação Interdisciplinar em Estudos do Lazer (PPGIEL). O Mestrado em Lazer da UFMG foi criado em 2006, e até hoje configura-se como o único programa voltado exclusivamente para tal temática. Já o Doutorado teve sua implementação autorizada pela CAPES no ano de 2012. Tais cursos são consequências da aproximação da UFMG com o lazer algumas décadas antes.

${ }^{1}$ A partir do ano de 2019, as atividades de dois grupos de pesquisa vinculados à Escola de Educação Física, Fisioterapia e Terapia Ocupacional da UFMG passaram a ser desenvolvidas em conjunto. Até então, o Polis era um grupo liderado pelo pesquisador Luciano Pereira da Silva e o NeoPolis coordenado pelas pesquisadoras Luciana Assis Costa e Daniete Fernandes Rocha. 
No ano de 1990, a professora Leila Mirtes de Magalhães Pinto criou o CELAR - Centro de Estudos do Lazer e Recreação na Escola de Educação Física, Fisioterapia e Terapia Ocupacional da UFMG (EEFFTO/UFMG), estrutura que foi responsável pelo desenvolvimento do campo do lazer na instituição. Tal fato ocorreu em decorrência de sua maior aproximação com essa temática, a partir de seu ingresso no curso de Mestrado em Educação Física da Universidade Estadual de Campinas - UNICAMP, sob orientação do Professor Antônio Carlos Bramante. Àquela época, a UNICAMP possuía grande destaque na produção do conhecimento no campo do lazer, oferecendo, inclusive, uma modalidade de curso de graduação específica para a área, o Bacharelado em Recreação e Lazer.

Atualmente, o PPGIEL oferece os cursos de Mestrado e Doutorado e está organizado em três linhas de pesquisa. Na linha 3, intitulada Formação, Atuação e Políticas do Lazer, concentramse as pesquisas que elegem como objeto as políticas públicas.

Dessa forma, através de diferentes ações, pesquisadores vinculados ao curso de Educação Física da UFMG e ao PPGIEL já desenvolvem, há pelo menos três décadas, estudos, pesquisas e ações no campo das políticas públicas de esporte e lazer. Parte dessas ações, até o ano de 2013, concentrava-se no Oricolé, Laboratório de Pesquisa Sobre Formação e Atuação Profissional em Lazer, liderado pelo professor Hélder Ferreira Isayama.

Ainda como uma Linha de Pesquisa do Oricolé, o Polis desenvolveu uma investigação intitulada Trajetória histórica das políticas públicas de esporte de Minas Gerais: 1938-2006² que teve como objetivo geral resgatar e analisar a trajetória histórica das Políticas Públicas de Esportes no período de 1938 a 2006, no estado de Minas Gerais. Tal ação foi financiada pela Fundação de Amparo à Pesquisa do Estado de Minas Gerais - FAPEMIG (Edital 16/2009) através de chamamento público que tinha o objetivo de apoiar ações científicas na área da História do Esporte e da preservação de acervos.

A expansão do corpo docente da EEFFTO, dentre outros fatores, pela política do REUNI Programa de Apoio a Planos de Reestruturação e Expansão das Universidades Federais, Decreto $n^{\circ}$ 6.096, de 24 de abril de 2007, possibilitou que novos pesquisadores ingressassem na instituição e no grupo de pesquisa.

A partir de 2013 o Polis tornou-se um grupo independente e estabeleceu uma parceria com a Secretaria Municipal de Esporte e Lazer - SMEL/PBH que, sensibilizada à época com a importância da memória, resolveu criar, inicialmente em suas instalações, o Centro de Memória do Esporte e do

${ }^{2}$ Publicação resultado desse projeto: RODRIGUES, Marilita Aparecida Arantes; ISAYAMA, Hélder Ferreira (Orgs.). Um olhar sobre a trajetória das políticas públicas de esporte e lazer em Minas Gerais: 19272006. Contagem: MJR, 2014. 
Lazer (CEMEL). Com isso, também financiada pela FAPEMIG, foi realizada uma pesquisa que procurou resgatar a memória das políticas municipais de esporte e lazer de Belo Horizonte. Esse tipo de investigação, constituiu-se em relevante possibilidade de fomento a debates e à compreensão da herança de vários processos construídos ao longo da história das políticas públicas de Belo Horizonte, que influenciaram e, ainda, influenciam a intervenção municipal.

Destaca-se também a participação do grupo na Rede Cedes - Centros de Desenvolvimento de Esporte Recreativo e de Lazer. Criada em 2003, a Rede Cedes desenvolveu, ao longo de sua história, diversas ações voltadas para a produção do conhecimento relacionado a políticas de esporte e lazer. O Polis integrou o Núcleo da Rede Cedes de Minas Gerais, implementado no ano de 2016. Nesse âmbito, desenvolveu investigação que elegeu como objeto o orçamento de Belo Horizonte para as áreas do esporte e do lazer ${ }^{3}$.

No ano de 2019, com a junção do Polis com o NeoPolis, amplia-se o escopo de investigação do grupo nos estudos de análise de políticas públicas com a inserção de docentes com formação em sociologia e ciência política. Tal junção foi motivada sobretudo pela percepção de que as atividades dos dois grupos eram similares e que, unidos, haveria um maior potencial para o desenvolvimento de investigações interdisciplinares.

A partir da trajetória histórica brevemente descrita anteriormente, os pesquisadores vinculados ao grupo desenvolvem investigações que emergem dos seus temas de interesse e de propostas de discentes que ingressam no Programa de Pós-Graduação Interdisciplinar em Estudos do Lazer. Dessa forma, o grupo Polis/Neopolis congrega estudantes de graduação, mestrado e doutorado da UFMG e de outras instituições de ensino, contribuindo para a formação de professores e gestores críticos e atuantes no cenário da educação e das políticas públicas de esporte e de lazer no Brasil.

A integração dos pesquisadores mais experientes com os estudantes de graduação se dá através do estudo de temas relacionados às políticas públicas de esporte e lazer, inclusive com o objetivo de que tais reflexões contribuam para que os discentes elaborem projetos para o ingresso em programas de pós-graduação stricto senso.

Participaram do grupo alunos do curso de Educação Física (16), Turismo (2), Administração de Empresas (2), Ciências Contábeis (1), Gestão Pública (1) e Terapia Ocupacional (1) que, atualmente, atuam em Universidades Federais (UFMG, UFP e UFJF), em Institutos Federais

${ }^{3}$ Publicação oriunda dessa ação: SILVA, Luciano Pereira; PEREIRA, Brisa de Assis; ABADE, Natascha Stephanie Nunes; SARTORI, Ludmila Miranda. Orçamento municipal para políticas de esporte e lazer: o caso de Belo Horizonte/MG. In: COUTO, Ana Cláudia Porfírio; SILVA, Luciano Pereira da; ROQUE, Camila Evelin; ALVES, Glauce Teixeira (Orgs.). Políticas Públicas de Esporte e Lazer: Centro MG da Rede CEDES /- Belo Horizonte: Utopika Editorial, 2019. 
(IFRN, IFMS e IFMG), em Instituições Privadas de Ensino Superior (FACUNICAMP, Rede Pitágoras e Centro Universitário Estácio BH), em escolas da Rede de Educação Básica Estadual e Privada, além do Terceiro Setor. Vale destacar que o conjunto dos atores envolvidos já circulou para muitas outras áreas de atuação chegando a ocupar espaço inclusive no Ministério do Esporte, em Secretarias Estaduais (Minas Gerais) e Municipais de Esporte e Lazer (Recife e Belo Horizonte).

Sobre a produção acadêmica do grupo no período de 2013-2020, foram elaboradas 5 teses de doutorado, 14 dissertações de mestrado e 7 trabalhos de conclusão de curso, sendo a maioria vinculada ao Programa de Pós-Graduação Interdisciplinar em Estudos do Lazer da UFMG. Além disso, foram levantadas 35 publicações, sendo os anos de 2018 e 2019 o período que o grupo mais produziu. Em geral, podemos afirmar que nossos objetos de investigação situam-se no campo da análise de políticas públicas de esporte e lazer, com destaque para: implementação de políticas, financiamento de políticas, avaliação de programas/projetos e políticas de inclusão.

As formas de publicações são diversas, variando de relatórios de pesquisa, produções técnicas, livros, capítulos de livros, artigos, projetos de pesquisa, resumos e resumos expandidos e trabalhos completos publicados em anais de congressos na área das políticas públicas de esporte e lazer.

O número de trabalhos completos em anais de congressos, artigos em revistas acadêmicas e projetos de pesquisa foram os principais meios de difusão do conhecimento. As revistas que tiveram maior número de artigos publicados foram a Movimento (UFRGS) e a Licere (UFMG). Os projetos de pesquisa abordaram as seguintes temáticas: "Políticas públicas de esporte e lazer: trajetória histórica da Secretaria Municipal de Esporte e Lazer de Belo Horizonte (1973-2012)"; "Implementação da política de esporte e lazer no estado de Minas Gerais"; "Implementação dos convênios do Programa de Esporte e Lazer da Cidade - PELC"; "A engenharia institucional de implementação dos projetos emergenciais de esporte e lazer no estado do Rio de Janeiro" e "Implementação da política de esporte e lazer no município de Belo Horizonte para pessoas com deficiência".

Para além do desenvolvimento de pesquisas em diferentes linhas, o grupo realiza ações interdisciplinares, de caráter transversal e interinstitucional, por meio de parcerias com diferentes grupos da comunidade científica, com o poder público e, ainda, com grupos internacionais.

As parcerias com a comunidade científica estimulam diálogos e debates que possibilitam o aprofundamento de uma reflexão crítica sobre as temáticas que envolvem o lazer, a educação física e as políticas públicas, além do desenvolvimento de pesquisas, publicações e realizações de eventos científicos e ciclos de debates. Entre essas parcerias podemos citar, para além das mencionadas 
anteriormente: 1) a ANPEL - Associação Brasileira de Pesquisa e Pós-Graduação em Estudos do Lazer: criada em 2013, congrega pesquisadores das mais diferentes áreas de conhecimento, que se dedicam à investigação do lazer e temas afins, a partir dos mais distintos pontos de vista teóricos e disciplinares; 2) o CBCE - Colégio Brasileiro de Ciências do Esporte: criado em 1978, é uma entidade científica que congrega pesquisadores ligados à área de Educação Física/Ciências do Esporte. Possui representações em vários órgãos governamentais, é ligado à Sociedade Brasileira para o Progresso da Ciência e está presente nas principais discussões relacionadas à essa grande área de conhecimento; 3) OTIUM - Lazer, Brasil e América Latina: idealizado em 2006 por docentes, pesquisadores e estudantes vinculados ao CELAR (Centro de Estudos de Lazer e Recreação) da EEFFTO/UFMG, que visavam ampliar os diálogos e a troca de experiências no contexto latino-americano. O grupo realizou em 2007, através do projeto "Lazer na América Latina/Tiempo libre, ocio y recreación en Latinoamérica", os primeiros estudos sobre o lazer na América Latina envolvendo autores latino-americanos de oito países.

A aproximação com o poder público se deu, por sua vez, com a Secretaria do Estado de Minas Gerais, via Subsecretaria de Estado de Esportes (SUBESP), vinculada à Secretaria de Estado de Desenvolvimento Social (SEDESE). Através do Observatório do Esporte de Minas Gerais, a SUBESP formulou, no ano de 2019, um documento intitulado "Propostas de ações conjuntas com Instituições de Ensino Superior" visando estratégias e parcerias de trabalho entre o estado e a academia. Esta parceria, possibilitou a ampliação do conhecimento acerca das ações do estado frente ao problema da exclusão social e acesso aos direitos sociais, além de evidenciar o interesse do estado nas produções acadêmicas que envolvam a Gestão Esportiva em suas diferentes frentes. A parceria vem possibilitando, ainda, o trânsito de servidores do estado para o interior da academia, através da participação em disciplinas eletivas voltadas ao estudo das Políticas Públicas, ofertadas pelo Programa de Pós-Graduação Interdisciplinar em Estudos do Lazer.

Por fim, quanto às parcerias internacionais, podemos citar o Departamento de Saúde Pública da Sheffield Hallam University (Inglaterra) e o Departamento de Ciências Sociais da Universidade Nova de Lisboa (Portugal). Essa internacionalização permite o conhecimento, o estudo e a troca de experiência em políticas públicas desenvolvidas em diferentes contextos culturais, econômicos e sociais, além da possibilidade de intercâmbio acadêmico de discentes e docentes.

O grupo Polis/Neopolis realiza reuniões quinzenais entre seus integrantes. Nelas, são discutidos temas que subsidiam as pesquisas desenvolvidas e promovem a formação dos pesquisadores (reuniões de estudo), são apresentados resultados parciais de pesquisas em desenvolvimento (principalmente de mestrandos e doutorandos) e são planejadas ações relacionadas a pesquisas coletivas. Tais ações, geralmente são de responsabilidade de duplas ou trios de 
pesquisadores, que se organizam de modo que cada grupo menor congregue pesquisadores mais experientes e iniciantes. Desde o segundo semestre de 2020, no contexto da pandemia de Covid-19 que impossibilitou ações presenciais, o grupo também tem adotado reuniões remotas com convidados externos que debatem temas de interesse do grupo.

Na próxima seção será apresentada uma breve síntese do arcabouço teórico que tem sustentado as discussões e produções do grupo de pesquisa sobre políticas de esporte e lazer. Optouse para este ensaio pelo recorte sobre o tema de implementação de políticas públicas.

\section{Síntese de abordagens de Implementação de políticas públicas}

A maioria dos estudos de políticas públicas de esporte e lazer trata prioritariamente de implementação e de avaliação. Todavia, ainda é comum o uso do termo implementação desarticulado da literatura da área de políticas públicas, tal como já evidenciado por Faria (2012) e Lotta (2019) em outras políticas setoriais. Vale ressaltar que a área de análise de políticas públicas surge ainda nos anos de 1950, e tem uma produção vasta em diferentes campos do conhecimento, sobretudo na sociologia, ciência política, administração pública e economia.

Os estudos de implementação de políticas públicas surgiram a partir da década de 1970 nos Estados Unidos, como uma reação às crescentes preocupações com a eficácia de amplos programas de reforma (PULZL \& TREIB, 2007). Conforme Lotta (2019), esses estudos buscam captar o que ocorre no momento de materialização ou concretização da política. Tais estudos remetem à perspectiva da política pública como um ciclo: agenda, formulação, implementação, avaliação, um instrumento analítico relevante, desde que ressalvado tratar-se de um modelo que não espelha necessariamente a realidade investigada, especialmente no que diz respeito às diferentes fases em sua inter-relação (LOTTA, 2019).

O campo de estudos sobre implementação de políticas públicas se desenvolve à vista das indagações sobre a diferença entre o que se planeja e o que é implementado de fato (ARRETCHE, 2001). Como afirma Arretche (2001), geralmente há uma grande distância entre os objetivos e o desenho concebido originalmente pelos formuladores das políticas públicas e a tradução de tais concepções em intervenções públicas. Com relação às análises do tema da implementação, podem ser apontadas quatro gerações de abordagem (LOTTA, 2019). Pode-se dizer que o ponto fulcral dessas distintas abordagens é a relação entre os objetivos das políticas e os seus resultados.

$\mathrm{Na}$ década de 1970, prevalecia a abordagem denominada top down, que se caracteriza pela visão de uma relação linear entre as metas políticas acordadas na fase de formulação e sua implementação. Assim, essa abordagem implicava o estabelecimento de procedimentos 
burocráticos adequados para garantir que as políticas fossem executadas com a maior precisão possível. Essa primeira geração provinha de preocupações iniciais em compreender os gaps entre o planejado e o executado, ou seja, o que acontecia quando as políticas públicas eram colocadas em prática e o que subvertia os resultados previamente desenhados. Destacam-se nessa abordagem: Pressman \& Wildavsky (1973), Van Meter \& Van Horn (1975), Bardach (1977), Sabatier \& Mazmanian (1979, 1980), bem como Mazmanian \& Sabatier (1983). Esses estudos tinham uma forte preocupação normativa baseada num ideal democrático de funcionamento do Estado (LOTTA, 2019, p. 16). A saída, portanto, seria aumentar a definição e clareza de objetivos e ampliar o controle de quem os executa.

Conforme Lima e D’Ascenzi (2019), na perspectiva top down, considera-se que a discricionariedade na implementação é intrínseca ao desenvolvimento de políticas, dado que a burocracia é percebida como "um ator fragmentado, com interesses próprios e capaz de promover visões específicas acerca das políticas públicas na quais atua" (HART \& WILLE, 2012). Logo, as agências implementadoras deveriam ter recursos suficientes à sua disposição para o funcionamento de um sistema de responsabilidades claras e controle hierárquico que supervisionasse as ações dos implementadores (PULZL \& TREIB, 2007).

Uma segunda geração de abordagem do tema da implementação de políticas públicas, no final da década de 1970 e no início da década de 1980, considera a perspectiva teórica do modelo bottom up, em contraposição ao modelo top down. Pesquisadores como Lipsky (1971, 1980), Elmore (1980) e Hjern (1982), entre outros, consideram que os resultados da política nem sempre se relacionam suficientemente com os objetivos políticos originais. Mas diferentemente da abordagem top down, os autores sugeriam que era necessário verificar o que ocorria no nível de entrega da política, começando com a identificação das redes de atores envolvidos. Atores envolvidos na implementação - como os street-level bureaucrats, ganham destaque na análise, apoiada em estudos da sociologia das organizações.

Ao longo do tempo acumularam-se críticas em ambas abordagens, considerando-se que a primeira não incorporava as complexidades dos processos e que a segunda enfatizava demasiadamente questões contextuais (BARRETT, 2004). Nos anos 1990, desenvolvem-se modelos alternativos de análise para além da discussão entre top down e bottom up (ELMORE, 1985; SABATIER, 1986), no sentido de combinar essas abordagens em uma terceira via, denominada "teorias híbridas". Segundo Pulzl e Treib (2007), as abordagens resumidas sob o título “teorias híbridas" tentaram superar a fragilidade do debate polarizado entre estudiosos das abordagens anteriores. A partir da perspectiva apoiada no Modelo Híbrido, o estudo da implementação de políticas públicas deve considerar o desenho institucional da política, entendido 
por meio do seu arcabouço normativo, bem como a estrutura organizacional e política do estado responsável por sua implementação.

Neste período também surge o modelo Advocacy Coalition Framework desenvolvido por Sabatier a partir de 1988, que busca compreender processos decisórios a partir e dentro das coalizões, no sentido de superar a contraposição entre formulação e implementação, abordando de forma contínua os processos decisórios que envolvem as políticas públicas e seus resultados (LOTTA, 2019).

Segundo Lotta (2019), atualmente os estudos de implementação encontram-se na quarta geração, que contempla várias formas de análise, fortemente orientadas pelos modelos de ação estatal advindos com os processos de reforma do Estado. Os temas que emergem nos atuais estudos de implementação colocam em evidência a questão da governança, a relação entre atores estatais e não estatais, os novos arranjos institucionais, os processos multiníveis, os sistemas de coordenação, as capacidades estatais na implementação, entre outros (LOTTA, 2019). Em síntese, os estudos têm se orientado pelo pressuposto de que a implementação de políticas públicas é influenciada por fatores relacionados a sistemas mais gerais (formato do estado, crenças e valores sociais, cultura nacional etc.), organizacionais e relacionados aos indivíduos que atuam nas políticas. Também tem se tornando consenso que o Estado não é mais o único ator envolvido nas políticas, uma vez que os arranjos de governanças são cada vez mais complexos (LOTTA \& VAZ, 2015; HUPE \& HILL 2002).

\section{Análise de Implementação à luz da quarta geração de estudos: federalismo, descentralização e sistema de coordenação intergovernamental}

As transformações ocorridas na sociedade brasileira pós-1988 mobilizaram o interesse pela área de políticas públicas, principalmente no que diz respeito às questões de centralização e descentralização e às propostas de reforma do Estado. É possível inferir que a Reforma do Estado colaborou para a construção e formação do campo de políticas públicas no Brasil (BRASIL \& CAPELLA, 2016). A descentralização do poder, a aposta na maior autonomia e responsabilização dos entes Federativos - com destaque para o Municipal - unidas à questão da maior democratização já indicadas na Constituição, surgem como um ponto importante nos estudos das políticas públicas no Brasil pós-redemocratização. Celina Souza (2007), assim como Farah (2011), refere-se ao processo da redemocratização brasileira e suas mudanças institucionais como fatores propulsores do crescimento do campo das políticas públicas.

A quarta geração de estudos de políticas públicas, por sua abordagem que abarca a complexidade desse fenômeno, orienta de uma forma mais ampla a análise do processo de 
implementação das políticas. No caso brasileiro, destacam-se, como relevantes, as questões relacionadas às particularidades do sistema federativo. Indaga-se como seus arranjos institucionais impactam na implementação de políticas, especialmente as políticas sociais. Abrucio e Franzese (2007) argumentam que o desenho institucional adotado no país é de suma importância para o entendimento sobre a produção de políticas públicas. Segundo os autores, dentre os fatores que mais influenciam na ação do Estado estão o sistema de governo e a organização territorial do poder. Acredita-se que são conceitos-chave para o entendimento de como o Estado, imerso em seus arranjos institucionais, tem viabilizado a implementação de políticas de esporte e lazer na extensão do território brasileiro.

\section{Federalismo e descentralização}

O Brasil adotou o modelo federalista de organização do Estado há mais de um século e essa divisão territorial de governo foi se modificando ao longo dos anos até chegar ao modelo vigente. Embora inspirado pelos moldes estadunidenses, existe uma peculiaridade, uma vez que o federalismo brasileiro não foi produto de um pacto entre os estados, como ocorreu nos Estados Unidos, e sim uma descentralização advinda do governo central para os governos locais.

O Federalismo é um sistema baseado na distribuição territorial - constitucionalmente definida e assegurada de poder e autoridade entre instâncias de governo, de tal modo que os governos nacionais e subnacionais são independentes em sua esfera própria de ação (ALMEIDA, 2005; MACHADO \& PALOTTI, 2015). Em sua forma original, o federalismo se caracteriza pela não centralização, pela difusão dos poderes governamentais entre muitos centros, nos quais a autoridade não resulta da delegação de um poder central. A existência de competências comuns entre instâncias de governo é a expressão mais clara da natureza não centralizada do federalismo. Todavia, as relações intergovernamentais variam muito entre os diversos países que o adotaram, bem como ao longo do tempo em um mesmo sistema federal (ALMEIDA, 2005).

Do ponto de vista das relações entre esferas de governo, a literatura distingue três tipos de arranjos federativos. O primeiro, chamado federalismo dual, constitui o modelo originário, no qual os poderes do governo geral e dos estados constituem soberanias distintas, que atuam de forma separada e independente, nas esferas que lhes são próprias (ALMEIDA, 2005). Os dois outros, nomeados de federalismo centralizado e federalismo cooperativo, são analisados como resultantes da transformação do arranjo dual, como consequência da tendência universal à expansão do escopo do governo federal, isto é, de um processo mais ou menos acentuado de centralização.

Os dois últimos tipos refletem relações intergovernamentais nas quais a não centralização, característica do ordenamento federativo, convive de forma complexa e, frequentemente, 
conflitante, com a lógica da centralização - descentralização (ALMEIDA, 2005).

No caso brasileiro, a $\mathrm{CF} / 88$ desenha um modelo federativo com traços de centralização, concentrando no nível federal a prerrogativa de legislar sobre a maior parte das políticas e delegando aos estados e municípios a responsabilidade por executá-las. A CF/88 instituiu a descentralização política e fiscal, elevando os municípios à categoria de entes federados e ampliando a transferência de receitas tributárias aos níveis sub-nacionais, conforme (BRASIL, 1988).

Em decorrência, o federalismo brasileiro pós constituinte foi marcado por duas tendências opostas: ao mesmo tempo em que houve um movimento de transferência de recursos financeiros para os níveis subnacionais - as transferências intergovernamentais, criadas ainda nos governos militares, e o aumento das competências dos entes federativos -, houve, também, a manutenção das competências legislativas do governo federal, acompanhada de uma grande capacidade para constranger a atuação dos estados e municípios (ARAÚJO, 2015).

Arretche (2004) acrescenta que, desde a primeira Constituição Federal, no ano de 1891, o Brasil optou pelo regime de separação de fontes tributárias, deixando claro quais impostos seriam de competência da União e quais seriam de competência dos estados. Para a autora, por mais que as competências tenham sido revistas ao longo do tempo, as mudanças mais bruscas ocorreram no período do regime militar, uma vez que houve uma centralização das competências, e, em seguida, no processo de redemocratização, com a Constituição de 1988, pautada pela descentralização fiscal.

Existem indícios de que, no caso brasileiro, as políticas públicas reguladas influenciam no sentido de redução dos patamares de desigualdade. Um estudo realizado por Arretche (2013) apresenta sólidas evidências de que o modelo centralizado do federalismo brasileiro tem sido um promotor da redução das desigualdades entre os entes federados.

Após a redemocratização, houve uma crescente demanda de serviços públicos, no contexto de demandas sociais, o que fez com que a relação entre os diferentes níveis de governo (União, estados, municípios e Distrito Federal) se tornasse ainda mais complexa (ABRUCIO \& FRANZESE, 2007). De fato, a CF/88 não definiu com clareza uma hierarquia de competências entre os níveis da federação, mas sim multiplicou as funções concorrentes entre União, estados e municípios, especialmente na área social (ALMEIDA, 1995:2005). O modelo de federalismo adotado no país prevê competências comuns a todos os níveis de governo com relação à provisão de políticas sociais, acentuando o problema da definição de responsabilidades entre entes federados marcadamente assimétricos (PALOTTI; COSTA, 2011; MACHADO; PALOTTI, 2015).

Arretche (2004) problematiza o fato de que a maior parte das políticas sociais brasileiras passou a ser competência de todos os entes federados, pois, segundo a autora, todos os níveis de 
governo são autorizados a implementar programas sociais (saúde, educação, assistência social, lazer, habitação, dentre outros), porém, não há obrigatoriedade de sua realização.

Ao longo da década de 1990, os diferentes graus de descentralização entre as políticas sociais estiveram dependentes ainda de características dessas próprias políticas, da heterogeneidade estrutural dos governos locais e dos arranjos institucionais constituídos (ALMEIDA, 1995; FARAH, 2001).

Somente no âmbito das reformas dos anos 1990 foram definidas mais claramente as responsabilidades federativas em matéria de política social, balizadas principalmente pelos instrumentos de coordenação definidos pelo governo federal. Segundo Arretche (2004) novas regras - introduzidas por meio de legislação ordinária, emendas constitucionais ou normas ministeriais, de acordo com cada política - foram necessárias para estimular a descentralização da execução das políticas sociais, não sendo suficiente ou auto-executável o princípio da descentralização presente na Constituição (ARRETCHE, 2009; BICHIR, 2011).

A descentralização das políticas sociais em contextos federativos evidencia a necessidade de compatibilizar a autonomia dos entes federados e a coordenação política para atingir objetivos comuns (PALOTTI; COSTA, 2011). O desenho das relações intergovernamentais e de mecanismos e estratégias de indução por parte das esferas mais abrangentes de governo são essenciais para determinar os resultados da descentralização de políticas sociais entre níveis de governo, no caso brasileiro.

Para que as políticas públicas alcancem um resultado considerável é necessário que exista uma coordenação central capaz de instruir os governos locais sobre como atuar em determinada política social. Os constrangimentos legais, incentivos financeiros e as parcerias são formas de coordenação para a execução de objetivos determinados por um plano nacional (MACHADO \& PALOTTI, 2015).

As discussões sobre federalismo e descentralização, sintetizadas nessa seção, têm subsidiado pesquisas desenvolvidas pelo Polis/NeoPolis. Por exemplo, estudo do grupo, cujo resultado foi submetido à Revista Sociologia Política (2020), objetivou analisar a implementação dos convênios do Programa Esporte e Lazer da Cidade no período de 2013 a 2017 em âmbito nacional, com foco nas possibilidades de continuidade do programa na esfera municipal. Para isso, buscou-se averiguar o potencial de indução e de coordenação do governo federal em relação aos municípios implementadores. 


\section{Relações intergovernamentais e coordenação das políticas}

Embora a $\mathrm{CF} / 88$ tenha transferido uma série de competências de execução de políticas para os municípios, também garantiu ao governo federal um grau de autoridade regulatória para influir na agenda política dos governos subnacionais (ARRETCHE, 2012).

Diante da heterogeneidade da capacidade de respostas dos municípios, e na tentativa de construção de mediações entre governo federal e entes subnacionais, verifica-se a tendência ao fortalecimento do governo federal no papel de coordenador de políticas e diretrizes nacionais a serem implementadas pelos municípios e estados, numa lógica de padrões mínimos de atuação vinculados a repasse de recursos com incentivos e induções, como já previa a própria Constituição (ALMEIDA, 2005; ARRETCHE, 2012; LOTTA, 2015). Esse movimento tem se justificado pelo fato de que, a partir do processo de descentralização, e dada a heterogeneidade das capacidades financeiras, técnicas e institucionais dos municípios, a provisão de políticas sociais tem se dado de forma desigual, e, amiúde, aquém das necessidades da população. Isso teria levado a um aumento da dependência dos municípios em relação ao governo federal (LOTTA; VAZ, 2015).

Arretche (2004) ressalta que embora os entes federados, no caso do Brasil, apresentem autonomia e independência dos três níveis de governo, nas áreas das políticas sociais, é fundamental que exista uma coordenação central capaz de orientar os governos locais em prol do atendimento às demandas da sociedade.

É preciso compreender que a municipalização não ocorre considerando-se somente os atributos estruturais das unidades locais de governo, como sua capacidade fiscal, administrativa e cívica. Arretche (1999) aponta que este processo se altera de acordo com requisitos institucionais das políticas a serem assumidas pelos governos locais, tais como o legado das políticas prévias, as regras constitucionais e a engenharia operacional de cada política social. No entanto, a ação política deliberada, ou seja, estratégias de indução eficientemente desenhadas para delegar a outro nível de governo a responsabilidade pela gestão destas políticas, pode compensar obstáculos à descentralização derivados de fatores de natureza estrutural ou institucional (UNGHERI, 2019). Assim, tanto os aspectos de coordenação da política quanto os de sua implementação assumem relevância, por envolverem o nível do governo federal e o dos governos locais.

Segundo Arretche (2004), a coordenação das políticas sociais é mais complexa em Estados federativos, pela sobreposição de competências ou competição entre os entes federados. Soares e Machado (2018) ressaltam a importância de uma coordenação intergovernamental principalmente nas federações, uma vez que há um aumento de centros decisórios e a realização de políticas públicas depende da convergência das escolhas governamentais para um atingimento das metas e objetivos em prol do atendimento das demandas da sociedade. 
Os autores acrescentam que as relações intergovenamentais não seguem um padrão para todas as áreas de políticas públicas, ou seja, por mais que o modelo adotado para algumas áreas tenha muitas semelhanças, em outras, contam com padrões próprios de relações intergovernamentais. Os diversos modelos adotados por cada área de políticas públicas podem trazer comportamentos distintos no que diz respeito a serem mais ou menos cooperativos, competitivos ou conflitivos (SOARES; MACHADO, 2018).

Para tratar das relações intergovernamentais, Soares e Machado (2018) esclarecem que cooperação não é o mesmo que dizer que há uma harmonia completa entre os entes federados, mas que há interesses comuns e, inclusive, interesses exclusivos de cada ente. Os autores identificam duas formas de cooperação - entre entes do mesmo nível de governo (horizontal) e entre determinado governo local e o governo central (vertical). Sua principal função é maximizar os benefícios para uma coletividade maior, mesmo que haja custos determinados em um acordo prévio.

No que diz respeito aos mecanismos de coordenação, importa dizer que a vertical tem como uma das principais características os constrangimentos constitucionais e legais, que obrigam os governos locais a seguirem uma diretriz central em detrimento de sua liberdade de escolha. Outra característica importante da cooperação vertical são os incentivos financeiros, fator que pode ser considerado um instrumento eficaz para induzir os governos locais a aderirem aos programas federais em cada área de atuação. Por último, mais uma característica desse mecanismo de cooperação é a pactuação de compromissos em áreas intergovernamentais, quando são tratadas em uma única arena, com todos os níveis de governo, as principais diretrizes para a implementação das políticas sociais. Todas essas características determinam obrigações morais entre todos os entes federados (SOARES; MACHADO, 2018).

Arretche (2004) ressalta que as relações verticais no Brasil (governo federal com os estados e municípios e dos estados com os municípios) têm como peculiaridade a independência dos entes federados, uma vez que tanto os estados, quanto os municípios são entes autônomos. Sendo assim, é possível que determinem suas próprias agendas, que podem ou não estarem de acordo com a agenda da União. Ainda conforme a autora, no que diz respeito à área das políticas sociais, por mais que os estados e municípios tenham autonomia para estabelecer as suas próprias agendas, quando há um objetivo nacional, é necessário que haja formas de induzir os demais entes federados a realizarem ações visando os mesmos objetivos e com certo grau de padronização. Se não for assim, não é possível garantir que tais objetivos serão priorizados pelos governos locais e se estarão de acordo com as estratégias elaboradas pelo governo central. 
Entender a dinâmica de coordenação intergovernamental é de suma importância para a melhor compreensão acerca das políticas públicas sociais. Um estudo realizado por Soares e Machado (2018) apontou que o modelo federativo adotado pelo Brasil apresenta problemas e desafios para a produção de políticas públicas, em especial para as políticas sociais, como é o caso do esporte e do lazer. Os autores elencam alguns desafios, como os "aspectos pouco equitativos do federalismo fiscal, o papel dos estados na dinâmica federal tripartite, a capacidade dos municípios na implementação de políticas públicas e tantos outros" (SOARES \& MACHADO, 2018, p.106).

No que concerne ao aspecto do federalismo fiscal, que trata, também, dos repasses da União para os governos locais, o estudo de Arretche (2010) revela que, mesmo com as dificuldades distributivas das questões fiscais, o governo federal atua no Brasil de maneira a diminuir a desigualdade interjurisdicional e, ainda segundo a autora, se não fossem essas transferências, a provisão dos serviços públicos seria extremamente desigual, uma vez que as políticas sociais em determinadas regiões mais ricas seriam bem diferentes das ofertadas nas regiões mais pobres.

Para além das questões fiscais, a própria função regulatória federal gera impactos sobre as decisões dos governos locais, uma vez que, por meio de mecanismos institucionais, os entes federados são induzidos a agirem em prol de um objetivo nacional, com diretrizes que coordenam as ações dos demais níveis de governo. Porém, é importante salientar que, mesmo com essas amarras institucionais, produto da regulação federal, os governos locais têm autonomia para tomar decisões a respeito da sua atuação na execução de políticas públicas (ARRETCHE, 2010). Essas considerações são importantes, pois, cabe ressaltar que não há um sistema nacional que coordene as políticas públicas de esporte e de lazer no contexto brasileiro, diferente do que acontece com a saúde e assistência social, e esse é um ponto de partida para estudos nessa área.

Assim, os processos de coordenação de políticas públicas têm sido objeto de reflexão do Polis/NeoPolis. Foi desenvolvido, por exemplo, no âmbito do grupo, um estudo que problematizou a ausência de uma coordenação intergovernamental e de aparatos legais que definam as atribuições e responsabilidades dos entes federados para a condução das políticas públicas de esporte e lazer. Nesse estudo foi analisada a atuação do estado de Minas Gerais na condução das políticas de esporte e lazer entre os anos de 2012 a 2018. A análise foi pautada nas dimensões do financiamento das políticas, na estrutura burocrática e nas ações implementadas pelo estado (KHALIFA, 2019).

\section{Perspectivas futuras do grupo}

Além da necessidade de aprofundamento teórico no campo de análise de políticas públicas, o grupo de pesquisa tem como meta ampliar sua produção tanto nos periódicos nacionais como internacionais. O investimento nas parcerias internacionais também se constitui propósito do grupo, 
com o intuito de abrir campo de pesquisa, bem como espaço de intercâmbio entre os alunos de Mestrado e Doutorado. Ainda como meta busca-se pleitear editais de fomento de pesquisa para o desenvolvimento dos estudos do grupo.

Acredita-se que o grupo Polis/NeoPolis tem um importante papel na qualificação de atores que atuam ou que podem no futuro atuar no campo das políticas públicas de esporte e lazer. Nesse sentido, diversas ações de formação podem ser realizadas. Destacamos: disciplinas optativas de graduação oferecidas a diversos cursos da UFMG; disciplinas eletivas ofertadas a pós-graduandos; eventos online em formato de lives para a discussão de temas relacionados às políticas públicas de esporte e lazer; cursos de curta duração direcionados a gestores públicos; assessoria técnica ofertada a instituições governamentais e não governamentais que desenvolvem políticas públicas de esporte e lazer.

Pretende-se ainda, estreitar os contatos com os setores públicos em diferentes níveis de governo, que desenvolvem políticas de esporte e lazer, com o objetivo de realizar pesquisas que contribuam com o campo prático da política pública.

\section{Considerações finais}

O campo de análise de políticas públicas tem como característica a interdisciplinaridade, constituída por várias áreas do conhecimento e pela diversidade das políticas setoriais. Há uma evidência de que, apesar do crescimento do campo, sua produção tem ocorrido de forma setorializada e fragmentada, dificultando a consolidação de um arcabouço teórico sustentado por um núcleo comum de conhecimento. Esse gap teórico tem inviabilizado a realização de estudos comparativos entre as políticas setoriais brasileiras, o que possibilitaria um avanço na investigação sobre a ação do Estado, pela insuficiência de parâmetros de comparabilidade.

No caso específico da produção de políticas de esporte e lazer no Brasil, não obstante a expansão de publicações na última década, a área se depara com a mesma fragilidade teórica para fundamentar os seus estudos. O grupo de pesquisa Polis/NeoPolis tem investido em estudos de políticas públicas, ancorados na ciência política e sociologia, com o intuito de produzir um conhecimento de uma política setorial que coadune com uma produção mais ampla do campo de políticas públicas nacionais e internacionais. Acredita-se que o recorte escolhido sobre os estudos de implementação possa sustentar teoricamente as pesquisas desenvolvidas na área de esporte e lazer.

A partir do conjunto de ações descritas criticamente nesse artigo, acredita-se que o grupo Polis/NeoPolis tem contribuído para a formação de indivíduos que atuam no campo do lazer em diferentes perspectivas, que englobam desde a área acadêmica, principalmente em instituições 
públicas de ensino superior, até a coordenação de políticas públicas de lazer em estruturas governamentais.

\section{REFERÊNCIAS}

ABRUCIO, Luiz Fernando; FRANZESE, Cibele. Federalismo e Políticas Públicas: o impacto das relações intergovernamentais no Brasil. In: Maria Fátima Infante Araújo; Lígia Beira. (Org.). Tópicos de Economia Paulista para Gestores Públicos. 1 ed. Edições FUNDAP: São Paulo, 2007.

ALMEIDA, Maria Hermínia Tavares de. Recentralizando a federação?. Revista de Sociologia e Política, Curitiba, n. 24, pp. 29-40, 2005.

ALMEIDA, Maria Hermínia Tavares de. Federalismo e políticas sociais. Revista Brasileira de Ciências Sociais, 28 (10): pp. 88-108, 1995.

ARAÚJO, Marcos Filipe Nogueira de. O Federalismo brasileiro e a posição do município no estado federal a partir de 1988. (Trabalho de Conclusão de Curso - Monografia), Curso de Bacharelado em Ciências Jurídicas e Sociais Direito, Centro de Ciências Jurídicas e Sociais, Universidade Federal de Campina Grande - Sousa- Paraíba - Brasil, p. $78,2015$.

ARRETCHE, Marta Teresa da Silva. Continuidades e Descontinuidades da Federação Brasileira: de como 1988 facilitou 1995. Dados (Rio de Janeiro. Impresso), v. 52, p. 377-423, 2009.

ARRETCHE, Marta Teresa da Silva. Federalismo e Igualdade Territorial: Uma Contradição em Termos?. Dados (Rio de Janeiro), v. 53, n.3, pp. 587-620, 2010.

ARRETCHE, Marta Teresa da Silva. Democracia, federalismo e centralização no Brasil. Rio de Janeiro: Editora FGV/Editora Fiocruz, 2012.

ARRETCHE, Marta Teresa da Silva. Dossiê agenda de pesquisa em políticas públicas. Revista Brasileira de Ciências Sociais, São Paulo, v. 18, n. 51, pp. 07-09, fev. 2003.

ARRETCHE, Marta Teresa da Silva. Estado federativo e políticas sociais: determinantes da descentralização. Rio de Janeiro: Revan, 2000.

ARRETCHE, Marta Teresa da Silva. Federalismo e políticas sociais no Brasil: problemas de coordenação e autonomia. São Paulo em perspectiva, 18(2), pp. 17-26, 2004.

ARRETCHE, Marta Teresa da Silva. Financiamento federal e gestão local de políticas federais : o difícil equilíbrio entre regulação, responsabilidade e autonomia. Ciência e Saúde Coletiva, Rio de Janeiro, v. 8, n. 2, p. 331-345, 2003.

ARRETCHE, Marta Teresa da Silva. Políticas Sociais no Brasil: descentralização em um Estado federativo. Revista Brasileira de Ciências Sociais. 14(40), pp. 111-141, 1999.

ARRETCHE, Marta Teresa da Silva. Quando as instituições federativas fortalecem o Governo Central? In: HOCHMAN, Gilberto; FARIA, Carlos Aurélio Pimenta de (Org.). Federalismo e políticas públicas no Brasil. Rio de Janeiro: Editora FIOCRUZ, 2013.

BARRETT, Susan M. Implementation studies: time for a revival? Personal reflections on 20 years of Implementation Studies. Public Administration, 82(2), pp. 249-262, 2004.

BICHIR, Renata Mirandola. Mecanismos federais de coordenação de políticas sociais e capacidades institucionais locais: o caso do Programa Bolsa Família. Tese de Doutorado. Rio de Janeiro: Universidade do Estado do Rio de Janeiro, 2011. 
BRASIL, Felipe Gonçalves, CAPELLA, Ana Cláudia Niedhardt. Os Estudos das Políticas Públicas no Brasil: passado, presente e caminhos futuros de pesquisa sobre análise de políticas. Revista Política Hoje, v. 25, nº 1, pp. 71-90, 2016.

BRASIL. 1988. Constituição da República Federativa do Brasil. Brasília : Senado Federal.

CAVALCANTE, Pedro; LOTTA, Gabriela Spanghero. (Eds.). Burocracia de médio escalão: perfil, trajetória e atuação. Brasília, DF: Escola Nacional de Administração Pública, 2015.

ELMORE, Richard. Forward and backward mapping: reversible logic in the analysis of public policy. In: HAND, K.; TOONEN, T. A. J. (ed). Policy implementation in Federal and Unitary Systems. Dordrecht: Martinus Nijhoff, pp. 71-96, 1985.

FARAH, Marta Ferreira Santos. Administração pública e políticas públicas. Revista de Administração Pública, v. 45, n. 3, pp. 813-836, 2011.

FARAH, Marta Ferreira Santos. Parcerias, novos arranjos institucionais e políticas públicas no nível local de governo. Revista de Administração Pública, v. 35, n. 1, pp. 119-145, 2001.

FARIA, Carlos Aurélio Pimenta de. Implementação: ainda o "elo perdido" da análise de políticas públicas no Brasil? In: FARIA, Carlos A. P. (Org.). Implementação de políticas públicas: teoria e prática. Belo Horizonte: Editora PUC Mina, pp. 123-153, 2012.

GONÇALVES, Renata; LOTTA, Gabriela Spanghero; BITELMAN, Marina Farkas. A coordenação federativa de políticas públicas duas décadas após a Constituição Federal de 88. In: Encontro Nacional de Administração Pública e Governança, 3, Salvador. Anais. Salvador, EnAPG, 2008.

GUNN, Lewis A.; HOGWOOD, Brian W. Models of policy-making centre for the study of public policy. Strathclyde: University of Strathclyde, 1982.

HART, Paul't; WILLE, Anchrit. Bureaucratic politics: opening the black box of executive government. In: PETERS, B. G.; PIERRE, J. The SAGE Handbook of public administration. London: Sage, pp. 328-339, 2012.

HILL, Michael; HUPE, Peter L. Implementation Public Policy. London: Sage Publications, 2002.

KHALIFA, Mehrez Palumbo. Atuação do estado de Minas Gerais nas políticas públicas de esporte e de lazer no período de 2012 a 2018: efeitos práticos frente à ausência de uma coordenação intergovernamental na área. Dissertação de Mestrado. Belo Horizonte: Universidade Federal de Minas Gerais, 2019.

LIMA, Luciana Leite; D’ASCENZI, Luciano. Implementação e Avaliação de Políticas Públicas: intersecções, limites e continuidades. In: LOTTA, G. S. (Org). Teoria e Análises sobre Implantação de Políticas Públicas no Brasil. Brasília: Enap, 2019.

LOTTA, Gabriela Spanghero. Burocracia e implementação de políticas de saúde. Rio de Janeiro: Editora Fiocruz, 2015.

LOTTA, Gabriela Spanghero. A política pública como ela é: contribuições dos estudos sobre implementação para a análise de políticas públicas. In G. Lotta (Org). Teoria e análises sobre implantação de políticas públicas no Brasil, Enap: Brasília, pp. 11-38, 2019.

LOTTA, Gabriela Spanghero; VAZ, José Carlos. Arranjos Institucionais de Políticas Públicas: aprendizados a partir de casos do Brasil. Revista Do Serviço Público, v. 66, n.2, pp. 171-194, 2015. DOI:

https://doi.org/10.21874/rsp.v66i2.409

MACHADO, José Ângelo; PALOTTI, Pedro Lucas de Moura. Entre cooperação e centralização: Federalismo e políticas sociais no Brasil Pós-1988. Revista Brasileira de Ciências Sociais, v. 30, n. 88, p. 61, 2 jun. 2015.

MINAS GERAIS. Secretaria de Estado de Desenvolvimento Social (SEDESE). Propostas de ações conjuntas com instituições de ensino superior. Subsecretaria de Estado de Esportes (SUBESP). Observatório do Esporte, p.11, 2019. 
PALOTTI, Pedro Lucas de Moura; COSTA, Bruno Lazzarotti Diniz. Relações intergovernamentais e descentralização: uma análise da implementação do Suas em Minas Gerais. Revista de Sociologia Política, Curitiba, v. 19, n. 39, pp. 211-235, jun. 2011.

PRESSMAN, Jeffrey L; WILDAVSKY, Aaron. Implementation. Berkeley: University of California Press, 1973.

PULZL, Helga; TREIB, Oliver. Implementing public policy. In: F. Fischer; G. J. MILLER; M. S. Sidney, eds.

Handbook of public policy analysis: theory, politics, and methods. Boca Raton: Taylor \& Francis Group, pp. 89-108, 2007.

RODRIGUES, Marilita Aparecida Arantes; ISAYAMA, Hélder Ferreira (Orgs.). Um olhar sobre a trajetória das políticas públicas de esporte e lazer em Minas Gerais: 1927-2006. Contagem: MJR, 2014.

SABATIER, Paul Armand. Top-Down and Bottom-Up Approaches to Implementation Research: a critical analysis and suggested synthesis. Journal of Public Policy, v. 6, n. 1, pp. 21-48, 1986. DOI:

https://doi.org/10.1017/S0143814X00003846.

SABATIER, Paul Armand; MAZMANIAN, Daniel A. de. The implementation of regulatory policy: a framework of analysis. Davis: Institute of Governmental Affairs: University of California, 1979.

SILVA, Luciano Pereira; PEREIRA, Brisa de Assis; ABADE, Natascha Stephanie Nunes; SARTORI, Ludmila Miranda. Orçamento municipal para políticas de esporte e lazer: o caso de Belo Horizonte/MG. In: COUTO, Ana Cláudia Porfírio; SILVA, Luciano Pereira da; ROQUE, Camila Evelin; ALVES, Glauce Teixeira (Orgs.). Políticas Públicas de Esporte e Lazer: CENTRO MG da Rede CEDES /- Belo Horizonte: Utopika Editorial, 2019.

SOARES, Márcia Miranda; MACHADO, José Angelo. Federalismo e Políticas Públicas. Brasília: Enap, 2018.

SOUZA, Celina. A Introdução Políticas Públicas: uma revisão da literatura. Sociologias, Porto Alegre, ano 8, $\mathrm{n}^{\circ} 16$, pp. 20-45, 2006.

SOUZA, Celina. Estado da Arte da Pesquisa em Políticas Públicas. In: HOCHMAN, Gilberto; ARRETCHE, Marta; MARQUES, Eduardo. Políticas Públicas no Brasil. Rio de Janeiro, Fiocruz. 2007.

SUDANO, Andréia C.P; SOARES, Alessandra G.; VERGILLI, Guilherme E. O Debate sobre a Pesquisa em Políticas Públicas no Brasil a partir da análise dos trabalhos apresentados na ANPOCS: evolução e desafios. Paper apresentado no XXXIX Encontro Anual da ANPOCS. Caxambu, ANPOCS, 2015.

UNGHERI, Bruno Ocelli. Políticas Sociais de Esporte e Lazer: institucionalização e municipalização no contexto do Programa Esporte e Lazer da Cidade. Licere, 22(2), pp. 647-648, 2019.

\section{NOTAS DE AUTOR}

\section{AGRADECIMENTOS}

Agradecemos aos integrantes do Grupo de Pesquisa em Políticas Públicas de Esporte e Lazer Polis/NeoPolis, ao Programa de Pós-Graduação em Estudos do Lazer - EEFFTO/UFMG e ao Programa de Mestrado em Estudos da Ocupação - EEFFTO/UFMG, aos quais o grupo está vinculado.

\section{CONTRIBUIÇÃO DE AUTORIA}

Concepção do manuscrito: L. P. da Silva, L. A. Costa, D. F. Rocha

Coleta de dados: B. A. Pereira, N. S. N. Abade

Análise de dados: B. A. Pereira, N. S. N. Abade, M. M. Mendes, M. P. de Oliveira

Discussão dos resultados: M. M. Mendes, M. P. de Oliveira 
Produção do texto: L. P. da Silva, L. A. Costa, D. F. Rocha, B. A. Pereira, N. S. N. Abade, M. M. Mendes, M. P. de Oliveira

Revisão e aprovação: L. P. da Silva, L. A. Costa, D. F. Rocha

FINANCIAMENTO - Não se aplica.

CONSENTIMENTO DE USO DE IMAGEM - Não se aplica.

APROVAÇÃO DE COMITÊ DE ÉTICA EM PESQUISA - Não se aplica.

CONFLITO DE INTERESSES - Não se aplica.

LICENÇA DE USO

Os autores cedem à Motrivivência - ISSN 2175-8042 os direitos exclusivos de primeira publicação, com o trabalho simultaneamente licenciado sob a Licença Creative Commons Attribution Non-Comercial ShareAlike (CC BY-NC SA) 4.0 International. Esta licença permite que terceiros remixem, adaptem e criem a partir do trabalho publicado, desde que para fins não comerciais, atribuindo o devido crédito de autoria e publicação inicial neste periódico desde que adotem a mesma licença, compartilhar igual. Os autores têm autorização para assumir contratos adicionais separadamente, para distribuição não exclusiva da versão do trabalho publicada neste periódico (ex.: publicar em repositório institucional, em site pessoal, publicar uma tradução, ou como capítulo de livro), com reconhecimento de autoria e publicação inicial neste periódico, desde que para fins não comerciais e compartilhar com a mesma licença.

\section{PUBLISHER}

Universidade Federal de Santa Catarina. Programa de Pós-Graduação em Educação Física. LaboMídia - Laboratório e Observatório da Mídia Esportiva. Publicado no Portal de Periódicos UFSC. As ideias expressadas neste artigo são de responsabilidade de seus autores, não representando, necessariamente, a opinião dos editores ou da universidade.

\section{EDITORES}

Mauricio Roberto da Silva, Giovani de Lorenzi Pires, Rogério Santos Pereira.

\section{EDITORES ASSOCIADOS DA SEÇÃO TEMÁTICA}

Fernando Henrique Carneiro; Marcelo de Paula Melo

\section{REVISÃO DO MANUSCRITO E METADADOS}

João Caetano Prates Rocha; Keli Barreto.

\section{HISTÓRICO}

Recebido em: 10 de dezembro de 2020.

Aprovado em: 20 de abril de 2021 . 